\title{
Analysis of plate spin motion and its implications for strength of plate boundary
}

Takeshi Matsuyama ${ }^{1}$ and Hikaru Iwamori ${ }^{1,2^{*}}$

\begin{abstract}
In this study, we investigate the driving forces of plate motion, especially those of plate spin motion, that are related to the toroidal components of the global plate velocity field. In previous works, numerical simulations of mantle convection have been used to examine the extent to which toroidal velocity components are naturally generated on the surface, by varying key parameters, notably the rheological properties of plates and plate boundaries. Here, we take the reverse approach and perform analyses of observed plate motions, which show an increase in the toroidal/ poloidal ratio at high degrees of spherical harmonic expansion, as well as a rapid change in the plate spin rate and the estimated driving stress around a critical plate size of approximately $1000 \mathrm{~km}$. This quantitative relationship constrains the strength at plate boundaries to 3-75 MPa, which is consistent with several seismological observations, including those from the NE Japan arc associated with the 2011 Tohoku earthquake.
\end{abstract}

Keywords: Plate spin motion, Toroidal-poloidal component, Driving force, Plate boundary, Strength

\section{Background}

A unique feature of the Earth is active plate tectonics (Schubert et al. 2001), involving rigid plates that interact at "soft" boundaries, the nature of which allows the relative motion between plates. Significant differences between the physical properties of plates and plate boundaries (e.g., strength and rheological properties) are critical to plate dynamic motions and contribute to the "toroidal motions" of the plates (e.g., Bercovici 2003; Moresi and Solomatov 1998), especially the significant strike-slip and spin motions of the plates (e.g., Bercovici and Wessel 1994; Cadek and Ricard 1992; Hager and O'Connell 1978; Lithgow-Bertelloni and Richards 1993; O'Connell et al. 1991; Olson and Bercovici 1991). In the absence of such differences, plate motions would likely be dominated by "poloidal motions," i.e., velocity fields without vertical vorticity at the Earth's surface.

The high toroidal/poloidal kinetic energy ratio, which characterizes plate tectonics, has been used as a criterion to evaluate the accuracy of three-dimensional

\footnotetext{
*Correspondence: hikaru@jamstec.go.jp

${ }^{1}$ Department of Earth and Planetary Sciences, Tokyo Institute

of Technology, 2-12-1 Ookayama, Meguro-ku, 152-8551 Tokyo, Japan

Full list of author information is available at the end of the article
}

simulations of mantle convection that naturally reproduce the surface motions of plate tectonics (e.g., Richards et al. 2001; Tackley 2000a, b). However, the mechanism of the plates' motions is not well understood at present (Bercovici 2003; Lithgow-Bertelloni and Richards 1993). If plate boundaries were sufficiently "hard" (i.e., rigid) to suppress relative plate motions, then the toroidal motion could not be generated. As the strength of plate boundary decreases, an appreciable toroidal motion begins to occur (Zhong et al. 1998), indicating that the mechanical strength of plate boundaries plays a key role in the plate dynamics of the Earth. Accordingly, a number of forward numerical simulations have examined various physical properties of the plates and their boundaries to reproduce Earth-like surface motions (e.g., Foley and Becker 2009; Lenardic et al. 2006; Richards et al. 2001; Tackley 2000a, b; van Heck and Tackley 2008).

Here, we adopt a different approach: Based on the observations of plate motion, we attempt to find the relationship between the motions and the mechanical strength of plate boundaries. We begin with a toroidalpoloidal decomposition of plate motion, based on the latest plate configurations (Argus et al. 2011; Bird 2003), to capture the global features of the velocity field. Then, we examine the motions of individual plates, particularly

\section{Springer}


their spin motions, which may determine the constraints on the strength of plate boundaries, using a force balance approach.

\section{Toroidal-poloidal analysis}

First, we performed a spherical harmonic expansion of the plate velocity field (e.g., Hager and O'Connell 1978). Plate boundaries (which include a narrow deformation zone) are key to the characterization of plate motions (Bercovici 2003; Gordon 2000); therefore, we used the high-resolution plate model of Bird (2003) (PB2002), which includes 52 plates defined by topography, volcanism, magnetic anomalies, moment tensor solutions, and geodesy. Then, we prepared a detailed global dataset at a resolution of $0.1^{\circ}$, and based on a hotspot reference frame (Gripp and Gordon 2002), we calculated the toroidalpoloidal components for spherical harmonic degrees that were higher than those performed in previous studies ( $l=1000$; i.e., to a resolution of $\sim 20 \mathrm{~km}$ ), by developing a new code that utilizes the parity of spherical harmonics ("Additional file 1"). The ratio $\gamma(l)$ of the toroidal power $\sigma_{\mathrm{T}}(l)$ to the poloidal power $\sigma_{\mathrm{P}}(l)$ is calculated as

$$
\gamma(l)=\frac{\sigma_{\mathrm{T}}(l)}{\sigma_{\mathrm{P}}(l)}=\sqrt{\sum_{m} c_{l m} c_{l m}^{*}} / \sqrt{\sum_{m} b_{l m} b_{l m}^{*}},
$$

where $c_{l m}$ and $b_{l m}$ represent the toroidal and poloidal coefficients of the velocity fields, respectively, and the asterisk denotes the complex conjugate. Note that the first-degree term in the toroidal expansion corresponds to the net rotation of the lithosphere, which depends on an absolute reference frame; we therefore do not discuss this term in this study.

The result of the spherical harmonic expansion is shown in Fig. 1. Although O'Connell et al. (1991) argued that the ratio of the amplitude of the toroidal to poloidal spectra is nearly constant for $l<32$ (at $\sim 0.8$ ), our new result, applicable to higher harmonics, shows that the amplitude ratio is broadly trends toward higher values for $l>20$, which corresponds to a scale $<1000 \mathrm{~km}$, and is demonstrated by the approximate lines in Fig. 1.

To estimate the error of this result, caused by the uncertainties in plate motions, we calculated the toroidal/poloidal ratio of another plate model, NNR-MORVEL (Argus et al. 2011). The result is indicated by the red line in Fig. 1 and demonstrates that there is a little difference in the toroidal/poloidal ratio between the plate models, indicating that the error of plate motion in a plate model is negligible and the trend of toroidal/poloidal ratio in this analysis is robust.

The increase in the ratio of the amplitude of toroidal to poloidal spectra is probably the result of two factors: one is the systematic increase in toroidal motion, such as

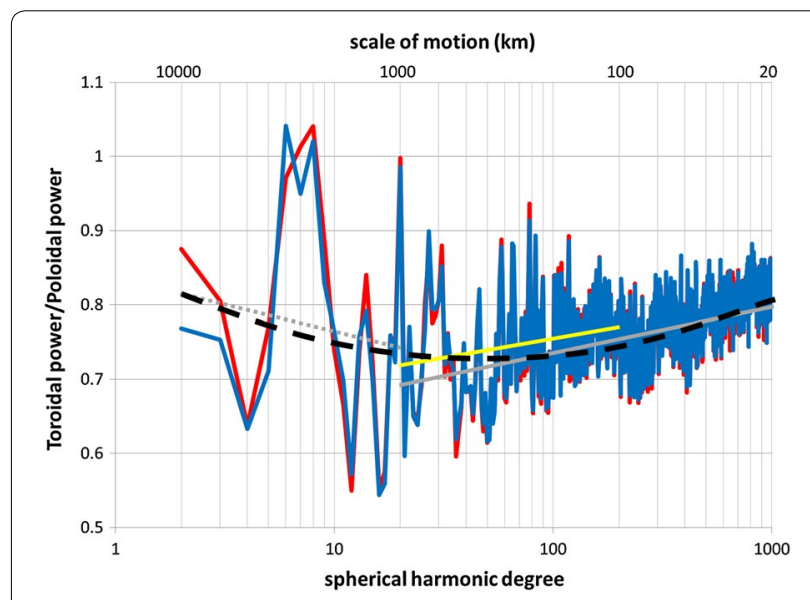

Fig. 1 Ratio of toroidal to poloidal power as a function of spherical harmonic degree. The blue and red spectra represent the ratios of the plate model from PB2002 (Bird 2003) and NNR-MORVEL (Argus et al. 2011), respectively. The upper horizontal axis shows the scale of motion corresponding to the spherical harmonic degree. There are four logarithmic trendlines to fit the data of PB2002: the broken black line is the quadratic fitting curve for the whole spectrum, the yellow line is the linear fitting line for the range from 100 to $1000 \mathrm{~km}$ in the scale of motion, and the gray dotted and solid lines represent linear fitting lines for $/<20(>1000 \mathrm{~km})$ and $/>20(<1000 \mathrm{~km})$, respectively. The range for the yellow line (100-1000 km) is important for the analysis of individual spin motion and the dynamics (Figs. 5, 6). The ratio of total toroidal power to total poloidal power, $\gamma$, is 0.752 for PB2002

plate spin motion, with decreasing plate size; the other is related to plate geometry, such as systematic changes in the aspect ratio of plates with varying plate sizes (Olson and Bercovici 1991). To quantitatively examine the effects of plate geometry and motion on the toroidal/poloidal ratio, we focused on individual plates and investigated variations in the geometries and motions of plates with variations in plate size.

\section{Analysis of individual plates}

To resolve the effects of plate geometry on toroidal/poloidal ratios, we quantified the aspect ratio of each plate with respect to its size. Hereafter, we analyze all the individual plates in the PB2002 data except for the plates dominated by active deformation zones that are expected to be inaccurate as a "rigid" plate in the plate model, such as the Balmoral Reef plate and the Aegean Sea plate. The size of each plate was represented by the diameter of a circle with an equivalent plate area. Olson and Bercovici (1991) demonstrated that the aspect ratio of a plate (i.e., the ratio of the plate length along the plate motion $L_{\mathrm{p}}$ to the plate length normal to the motion $L_{\mathrm{n}}$ ) has the following relationship with the toroidal/poloidal kinetic energy ratio $\gamma$ :

$$
\gamma \sim\left(\frac{L_{\mathrm{p}}}{L_{\mathrm{n}}}\right)^{2}
$$


Although Eq. (2) is obtained for a highly idealized geometry, this relationship is valid for slightly more generalized cases as shown in Fig. 2, in which the relationship

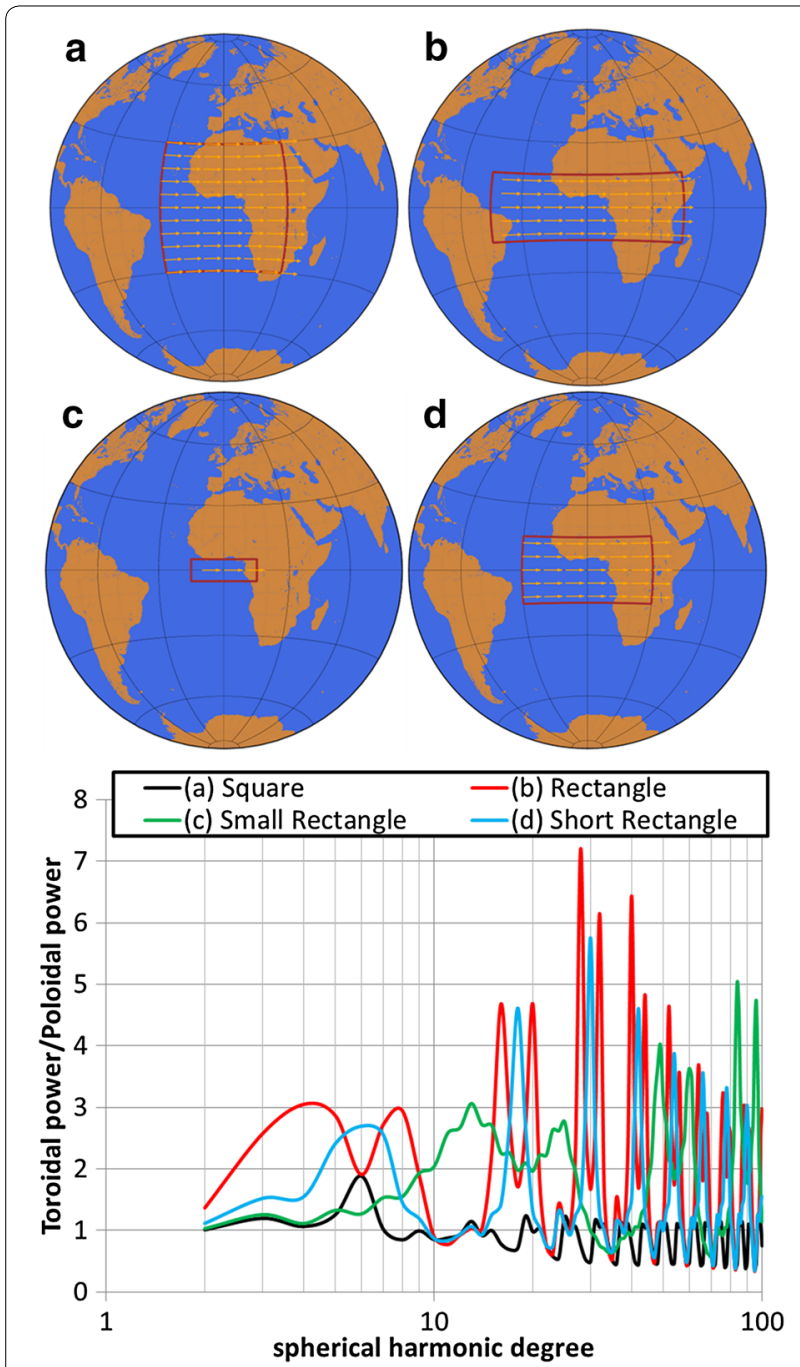

Fig. 2 Relationship between the plate size, aspect ratio, plate motion, and toroidal/poloidal ratio, based on several idealized cases. Top idealized plate boundary and its motion. All the plates have the same Euler pole at the North pole, and the induced eastward plate motions are represented by orange vectors. a a square plate $\left(60^{\circ} \times 60^{\circ}\right)$, b a rectangular plate $\left(30^{\circ} \times 90^{\circ}\right)$, c a small rectangular plate with the same aspect ratio as in $(\mathbf{b})\left(10^{\circ} \times 30^{\circ}\right)$ and $\mathbf{d}$ a rectangular plate with a smaller aspect ratio than $(\mathbf{b})$ and $(\mathbf{c})$ (i.e., closer to square, $30^{\circ} \times 60^{\circ}$ ). Bottom toroidal/poloidal (T/P) ratios for the plate motions of (a-d). Compared to the square plate (a) that has almost equal toroidalpoloidal power, i.e., T/P ratio $\sim$ 1, the rectangular plate $\mathbf{( b )}$ shows increased T/P ratios at higher spherical harmonic degrees (i.e., finer scale of motion). For the rectangular plate (d), the T/P ratio is smaller compared with (b), which suggests that the aspect ratio affects the amplitude of toroidal-poloidal spectra (a larger aspect ratio increases the T/P ratio). In addition, the small rectangular plate (c) shows significantly increased $T / P$ ratios at even higher spherical harmonic degrees compared with (b) and (d) between the aspect ratio, plate size and the toroidal/ poloidal spectra has been calculated and plotted. Figure 2 shows that the higher aspect ratio increases the toroidal/ poloidal ratio, especially at high degrees of spherical harmonics (b, c and d in Fig. 2), and such increased toroidal/poloidal ratios occur at higher spherical harmonic degrees (i.e., finer scale of motion) for the smaller plate (c in Fig. 2). Therefore, $\gamma$ in Eq. (2) can be regarded as a good measure to investigate the influence of plate geometry on the toroidal/poloidal ratio. Accordingly, we estimate the lengths of each plate in the directions parallel and normal to the observed plate motion. Figure 3 shows that although the aspect ratio of five plates exceeds 9 around the plate size of $1000 \mathrm{~km}$, the aspect ratio does not systematically vary with plate size, indicating that aspect ratio is not the primary cause of the systematic increase in the toroidal/poloidal ratio observed in Fig. 1.

Subsequently, we examined individual plate motions with respect to plate size. For the analysis, we divided plate motions into two types, spin motion and straight motion. The plate spin motion generates a shear motion at the plate boundary and is associated with toroidal motion, whereas straight plate motion results in poloidal motion at both subduction zones and ridges, and toroidal motion along transform faults. Therefore, the ratio of spin motion to straight motion can be used as an index of the toroidal/poloidal ratio.

To obtain the spin and straight motions of individual plates, we divided the Euler vector of each individual plate into two components: a vector that passes vertically through the geometric center of the plate, which is related to the spin motion and has a magnitude (i.e., angular velocity) defined as $\omega_{C}$, and a vector perpendicular to the first vector, which passes through the Earth's center and is related to the motion along a great

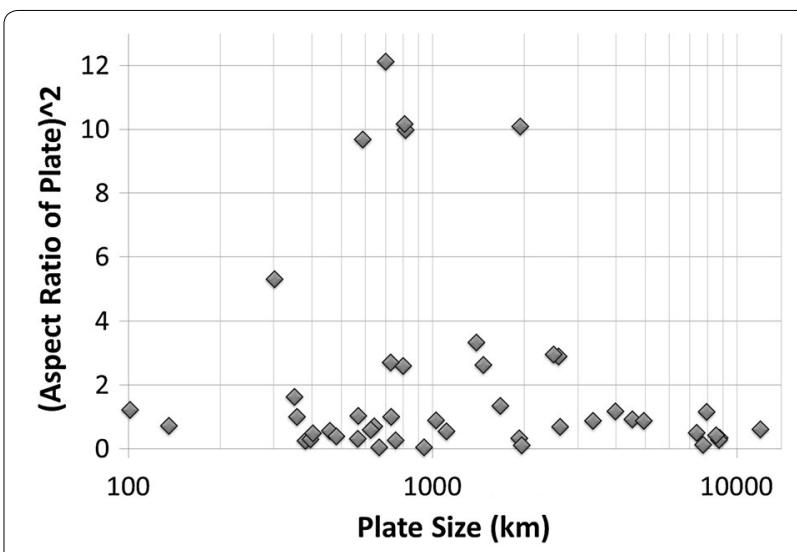

Fig. 3 Aspect ratio of each plate as a function of plate size. Note that small plates in this figure (left side) correspond to high spherical harmonic degrees in Fig. 1 (right side) 
circle, and whose magnitude is defined as $\omega_{G}$. For plates in the PB2002 dataset, plots of $\omega_{C}$ and $\omega_{G}$ as functions of plate size (Fig. 4) show that $\omega_{C}$ generally decreases with increasing plate size, whereas $\omega_{G}$ is roughly constant between 0.1 and $1 \%$ Myr. As a result, for plate sizes $<\sim 1000 \mathrm{~km}$, the difference between $\omega_{C}$ and $\omega_{G}$ is large (Fig. 4). This difference can induce variations in the toroidal/poloidal ratio at high spherical harmonic degrees (Fig. 1). Based on these differential variations, we discuss the mechanisms and their corresponding force balance for the plate motions.

\section{Dynamics of plate spin motion}

The driving forces of plate motion are generally classified into three types (e.g., Forsyth and Uyeda 1975): body forces (e.g., slab-pull and ridge-push; hereafter expressed as $T_{\mathrm{BD}}$ ), plate boundary forces (e.g., collision, suction, and shear forces between neighboring plates; $T_{\mathrm{PB}}$ ), and resultant resistance forces (e.g., mantle drag and slab resistance; $T_{\mathrm{RS}}$ ). Here, mantle drag (a component of $T_{\mathrm{RS}}$ ) is treated as a resistive force rather than a driving force of plate spin motion, because the toroidal components in mantle convection are negligible (Hager and O'Connell 1978) and are unlikely to excite the spin motion of plates. Of these, $T_{\mathrm{BD}}$, in particular the slab pull force, is the main driving force of the global plate system (Forsyth and Uyeda 1975); $T_{\mathrm{BD}}$ contributes primarily to straight plate motion, whereas $T_{\mathrm{PB}}$ may cause spin motion when torque occurs around the center of the plate. However, for the slab pull force, spin motion can be excited. One such

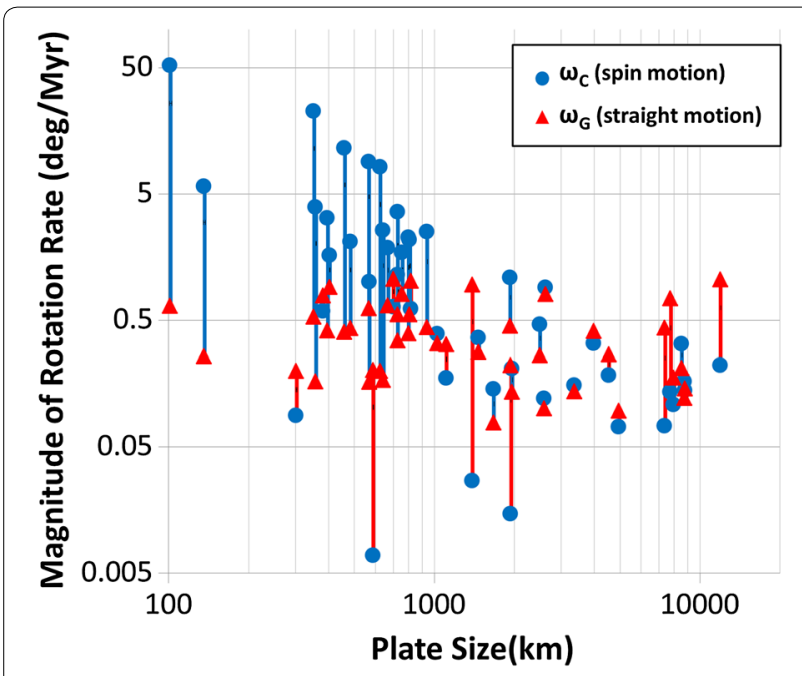

Fig. 4 Plate spin $\left(\omega_{C}\right)$ and straight $\left(\omega_{G}\right)$ motions as a function of plate size. Blue circles and red triangles represent $\omega_{C}$ and $\omega_{G}$, respectively. The vertical lines between blue circles and red triangles connect a $\omega_{C}$ , $\omega_{G}$ pair for each plate, and the color of the line indicates a larger component of either $\omega_{C}$ (blue lines) or $\omega_{G}$ (red lines) example is the Cocos plate. Gorbatov and Fukao (2005) have shown that the northwestern part of the slab was torn away from the deeper Farallon slab. It induces the heterogeneity of the slab pull forces, including a strong northward force from the eastern part of the slab, which can lead to the observed counterclockwise spin motion. Another example is the Philippine Sea plate that exhibits an active spin motion (Seno et al. 1993). Seno (2000) suggests that the spin motion results from the eastward mantle flow against the Philippine sea slab subducted beneath the SW Japan-Ryukyu arc, which is indicated by observations of electric conductivity (Handa 2005; Shimoizumi et al. 1997) and mantle anisotropy (Long and Hilst 2005). To exclude such complexities associated with slabs, we focused on plates without slabs, in which case the torque balance around the center of the plate can be described with relevant $T_{\mathrm{PB}}$ and $T_{\mathrm{RS}}$ forces, as discussed below. We can express $T_{\mathrm{PB}}$ as the driving shear stress along a plate boundary $\sigma_{\mathrm{PB}}$ and the area receiving the stress $S_{\mathrm{PB}}$ as

$$
T_{\mathrm{PB}}=\sigma_{\mathrm{PB}} S_{\mathrm{PB}} R=\sigma_{\mathrm{PB}} D_{\mathrm{PB}} L_{\mathrm{PB}} R=2 \pi \lambda \sigma_{\mathrm{PB}} D_{\mathrm{PB}} R^{2}
$$

where $R$ is the plate radius, $D_{\mathrm{PB}}$ is the average depth of the plate boundary sustaining the shear stress, $L_{\mathrm{PB}}$ is the length of the plate boundary along which the driving force is applied, and $\lambda$ is the ratio of $L_{\mathrm{PB}}$ to the total length of the plate boundary. In Eq. (3), we assume a planar plate for simplicity in the calculation of its radius and area (which does not affect the results significantly), and we can assume that the plate is circular rather than spherical shell because the difference in the result is not sufficiently significant, the length of the driving plate boundary is proportional to the plate size, and $\lambda$ is constant. We also assume the presence of a low-viscosity layer (hereafter referred to as the asthenosphere) with constant Newtonian viscosity $\mu$ and thickness $D_{\mathrm{RS}}$. Then, $T_{\mathrm{RS}}$ can be expressed as

$$
T_{\mathrm{RS}}=\int \sigma_{\mathrm{RS}} r \mathrm{~d} S=\int_{0}^{R} \mu \frac{r \omega_{C}}{D_{\mathrm{RS}}} 2 \pi r^{2} \mathrm{~d} r=\frac{\pi \mu \omega_{C}}{2 D_{\mathrm{RS}}} R^{4} .
$$

From the torque balance and Eqs. (3) and (4), we obtain

$$
\sigma_{\mathrm{PB}}=\frac{\omega_{C}}{4 \lambda D_{\mathrm{PB}} D_{\mathrm{RS}}} \mu R^{2},
$$

which indicates that $\sigma_{\mathrm{PB}}$ is proportional to $R^{2}$ and $\omega_{C}$. In other words, Eq. (5) shows that (for a constant $\omega_{C}$ ) as plate size increases (left to right in Fig. 4), the driving shear stress increases such that it induces spin motion onto a larger plate.

In this context, $\omega_{C}$ of a slab-free plate, which is indicated by the blue circles in Fig. 5, generally increases with decreasing plate size. One notable feature in Fig. 5 is the rapid change in $\omega_{C}$ around the critical plate size 
of $\sim 1000 \mathrm{~km}$ : above the critical size, $\omega_{C}$ values are generally less than the average rotation rate of the global lithosphere (i.e., the net lithospheric rotation; Ricard et al. 1991), of $\sim 0.43^{\circ} / \mathrm{Myr}$, as based on a hotspot reference frame; below the critical size, however, except for two plates, i.e., the Panama plate and the Shetland plate, the motions of which are not well determined in PB2002 model, all $\omega_{C}$ values exceed $0.43^{\circ} / \mathrm{Myr}$.

It should be noted that the $R-\omega_{C}$ variation is not significantly affected by the choice of different plate models as shown in Fig. 5 [i.e., Pb2002 (Bird 2003), NNR-MORVLE (Argus et al. 2011) and GSRM v2.1 (Kreemer et al. 2014) that include both no-net-rotation and hotspot reference frames for the data acquired by several methods representing different timescales. See "Additional file 2: Table S1" for details]: i.e., almost all the large plates (plate size $>\sim 1000 \mathrm{~km}$ ) show negligibly small $\omega_{C}$ less than the netrotation rate (0.43), and the rotation direction (clockwise or counterclockwise) may vary depending on the reference frame chosen (as indicated by large error bars with downward arrows in Fig. 5), whereas the large $\omega_{C}$ is seen only for small plates irrespective of the chosen frame (Fig. 5).

In order to test such a possibility, we examine the relationship between $R$ and $\sigma_{\mathrm{PB}}$ (the plate boundary shear

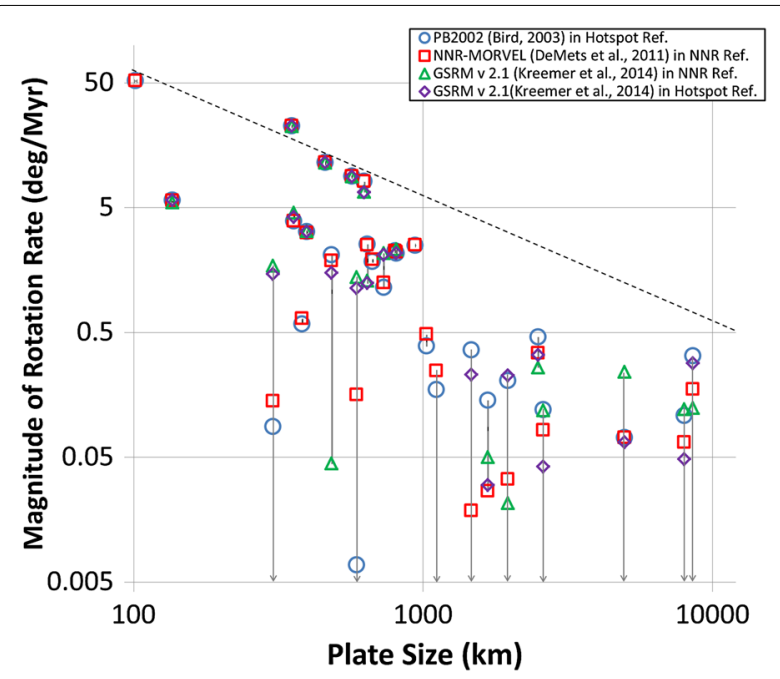

Fig. 5 Rotation rate and plate size. Blue circles, red squares, green triangles, and purple diamonds represent the data from three different models of PB2002 (Bird 2003), NNR-MORVEL (Argus et al. 2011), GSRM V2.1 (Kreemer et al. 2014) in no-net-rotation, and hotspot reference frame, respectively (see "Additional file 2: Table S1"for the details). Vertical bars represent the difference in rotation rate of a plate among the three plate models, and the downward arrows indicate that both clockwise and counterclockwise rotations occur depending on the plate models with different reference frames. The dashed line represents the expected rotation rate driven by the motion of the Pacific plate $(\sim 10 \mathrm{~cm} /$ year $)$ stress driving the spin motion) based on Eq. (5) and observed $\omega_{C}$ as shown in Fig. 6, assuming $\mu=10^{20} \mathrm{~Pa} \mathrm{~s}$, $D_{\mathrm{PB}}=40 \mathrm{~km}, D_{\mathrm{RS}}=60 \mathrm{~km}$, and $\lambda=0.25$. The uncertainties associated with these assumptions will be discussed later. It is worth noticing that $\sigma_{\mathrm{PB}}$ for plates with low rotation rates (the large plates, in general) have extremely large uncertainties, indicated by the error bars with downward arrows in Fig. 6, and should be regarded as upper bounds.

The dashed line in Fig. 6 represents the expected driving stress induced by the motion of the Pacific plate (corresponding to the dashed line in Fig. 5), which limits the upper bounds of $\sigma_{\mathrm{PB}}$ for the small plates. The small plates plot along the dashed line are located next to (or very close to) the fast-moving Pacific plate and are expected to have a high $\sigma_{\mathrm{PB}}$ as in Fig. 6, whereas other small plates located next to plates with slower velocities are expected to have a lower $\sigma_{\mathrm{PB}}$, which is also seen in Fig. 6. Within this context, in order to discuss the maximum stress that the plate boundary can sustain, the upper bound of $\sigma_{\mathrm{PB}}$ and its variation with plate size are thought to be more important than the overall data distribution. For large plates, $\sigma_{\mathrm{PB}}$ is appreciably lower than the prediction of the dashed line, indicating that rheological weakening or yielding operates on the boundaries of large plates, irrespective of the choice of reference frame (Fig. 6).

For small plates (the plate size $<\sim 1000 \mathrm{~km}$, i.e., $R<500$ $\mathrm{km})$, the shear stress driving the spin motion increases with $R$ (e.g., the dashed line in Fig. 6), and above a critical size $R_{\mathrm{c}}$, the stress becomes too large to transmit the stress

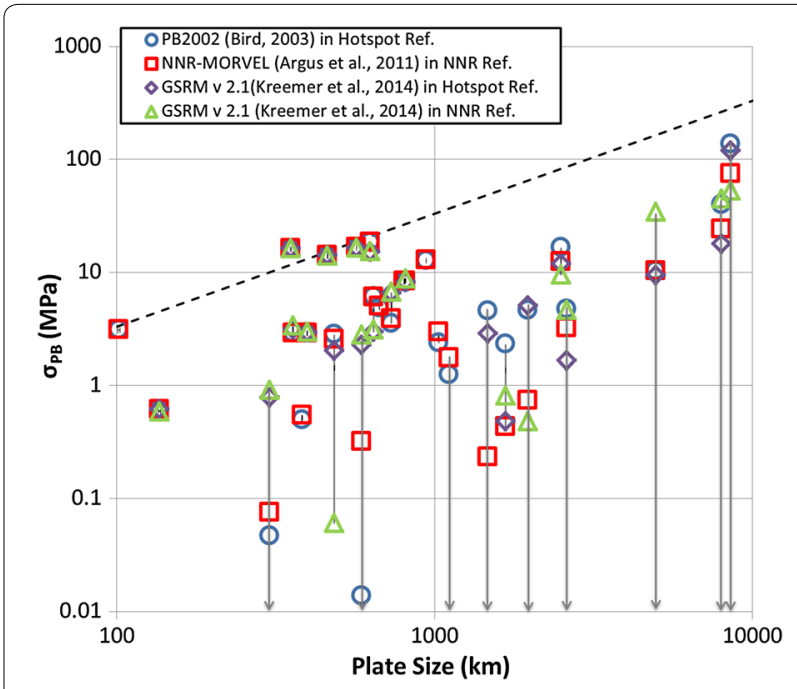

Fig. 6 The driving shear stress along a plate boundary $\sigma_{P B}$ obtained from Eq. (5) as a function of plate size $(=2 R)$. The symbols and error bars are the same as in Fig. 5. The dashed line is the expected driving stress induced by the motion of the Pacific plate (corresponding to the dashed line in Fig. 5) 
across the boundary, causing rheological weakening or yielding. This critical stress is regarded as the strength of the plate boundary. From Fig. 6, the critical stress is estimated to be $\sim 10-20 \mathrm{MPa}$ for plate sizes between $\sim 350-630 \mathrm{~km}$. Accordingly, the rotation rate of plates with $R>R_{\mathrm{c}}$ is small as compared to the dashed line in Fig. 5.

It can be confirmed that the spin rates of small plates are higher than those of large plates, which is the overall result, from Fig. 7, which shows that many small plates along the "sides" (i.e., along strike-slip boundaries) of large fast-moving plates have high spin rates with a rotation direction (clockwise or counterclockwise) consistent with the nearly straight motions of large plates that subduct along their margins. This mechanism has been suggested for several individual microplates (e.g., Schouten et al. 1993). Figure 7, for example, demonstrates that in the southwestern Pacific, the Pacific plate (PA) excites spin motion of the Niuafo'ou plate (NI) and that the Australia plate $(\mathrm{AU})$ induces spin motion of the Tonga plate (TO) and the Kermadec plate (KE). Along the East Pacific Rise, the Pacific plate and the Nazca plate (NZ) drive motions of the Easter plate (EA) and the Juan Fernandez plate (JZ). Although some of the abovelisted small plates, based on PB2002 plate model, are located within deforming zones identified by Kreemer et al. (2014) and could be inappropriate to consider them as rigid plates to define the spin rate (e.g., KE), the overall configuration remains unchanged, including large spin motions of NI, EA, and JZ as shown in Fig. 7.

These features, represented in Fig. 7, suggest that although some regions (especially Southeast Asia) exhibit complex spin directions probably due to interactions among the small plates, large fast-moving plates with subducting slabs induce spin motion in adjacent small plates through interactions along plate boundaries, which supports the idea presented above that $T_{P B}$ drives the plate spin motions.

In addition, from Eq. (5) and Fig. 6, we obtain a quantitative relationship between the viscosity of the asthenospheric mantle beneath the plates $\mu_{\mathrm{a}}$ and the strength of the plate boundary $\sigma_{\mathrm{st}}$. We substitute the constraints at the critical condition, as specified in Fig. 6 (i.e., $\omega_{C} \approx 8^{\circ}$ $/ \mathrm{Myr}$, plate size $\approx 630 \mathrm{~km}$ and $\lambda \approx 0.25$, corresponding to the South Bismarck plate attaining the maximum $\left.\sigma_{\mathrm{PB}}\right)$, and set $D_{\mathrm{PB}}=40 \mathrm{~km}$ as the thickness of the plate boundary sustaining the shear stress (e.g., Kohlstedt et al. 1995). Then, we obtain the following equation,

$$
\sigma_{\mathrm{st}}(\mathrm{MPa})=\frac{1.1}{10^{17}} \frac{\mu_{\mathrm{a}}(\mathrm{Pa} \mathrm{s})}{D_{\mathrm{RS}}(\mathrm{km})},
$$

which is the basis for Fig. 8. There is an appreciable uncertainty with regard to the thickness of the asthenosphere beneath the oceanic plates, as it is dependant on the observational methods used for measurement (Karato 2012); the asthenosphere is observed as a zone of low

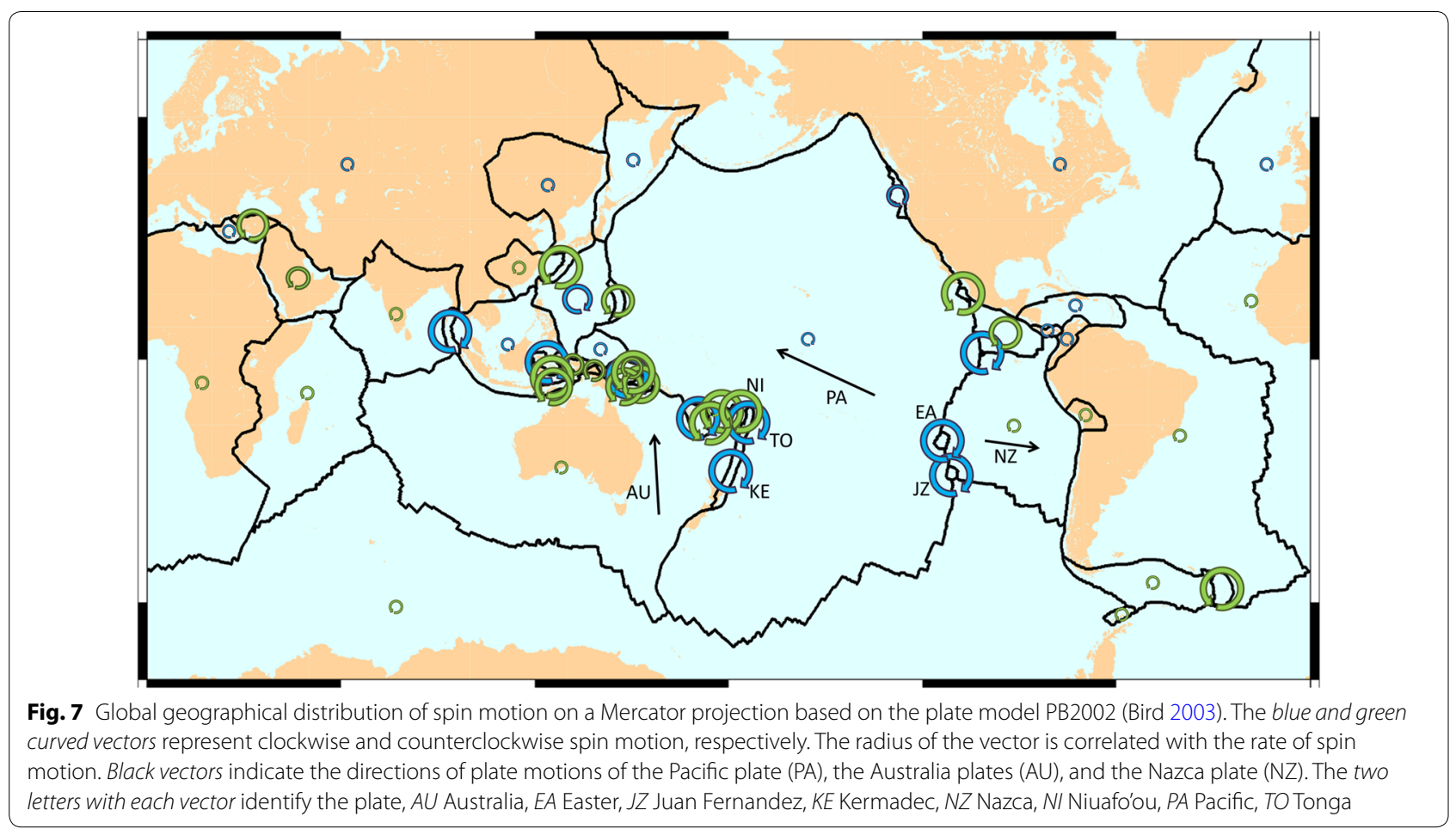




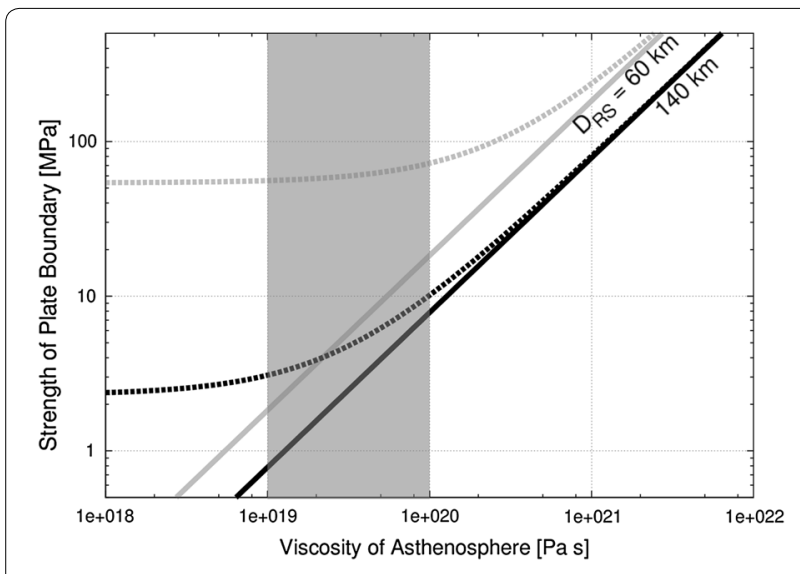

Fig. 8 Relationship between the strength of a plate boundary and the viscosity of the asthenosphere. The solid black and gray lines are obtained from Eq. (6) for $D_{R S}=140$ and $60 \mathrm{~km}$, respectively. The dotted black and gray lines are obtained on the basis of Eq. (7), corresponding to the minimum and maximum estimates for the strength of plate boundary, respectively. The shaded region shows the range of viscosity of the asthenosphere estimated from post-glacial rebound, seismic data, and experimental data (e.g., Forte and Mitrovica 2001; Karato and Wu 1993; Simons and Hager 1997)

seismic velocity [e.g., 120 km (Kawakatsu et al. 2009)], a high attenuation layer [e.g., $\sim 140 \mathrm{~km}$ (Dziewonski and Anderson 1981); 60 km (Yingjie et al. 2007)] with significant seismic anisotropy [e.g., $\sim 120 \mathrm{~km}$ (Beghein and Trampert 2004)] and a high electric conductivity layer [e.g., $\sim 60 \mathrm{~km}$ (Evans et al. 2005)]. From these observations, we estimate the thickness of asthenosphere under the oceanic plates ( $\left.D_{\mathrm{RS}}\right)$ to be $60-140 \mathrm{~km}$.

Equation (6) and Fig. 8 impose several constraints on the plate-mantle dynamics. Substituting $\mu_{\mathrm{a}}=10^{21} \mathrm{~Pa} \mathrm{~s}$, based on the representative viscosity of the upper mantle (Peltier 1998), into Eq. (6), we obtain $\sigma_{\text {st }}=78-183 \mathrm{MPa}$ for $D_{\mathrm{RS}} \approx 60-140 \mathrm{~km}$. Considering a more realistic case and assuming a low-viscosity asthenosphere, which is estimated from post-glacial rebound, seismic data, and laboratory measurements as $10^{19}-10^{20} \mathrm{~Pa}$ s (e.g., Karato and Wu 1993; Simons and Hager 1997; Forte and Mitrovica 2001 ), we substitute $\mu_{\mathrm{a}} \approx 10^{19}-10^{20} \mathrm{~Pa}$ s, which gives $\sigma_{\text {st }} \approx 0.78-18 \mathrm{MPa}$. This estimate only considers the force along the fractional length of the plate boundary $\lambda$, as in Eq. (3). If we consider the resistive forces along the remainder of the plate boundary, with length $1-\lambda$, we obtain

$$
\sigma_{\mathrm{PB}}=\frac{\omega_{C}}{4 \lambda D_{\mathrm{PB}} D_{\mathrm{RS}}} \mu R^{2}+\frac{1-\lambda}{\lambda} \sigma_{\mathrm{R}},
$$

where $\sigma_{\mathrm{R}}$ is the average resistive stress along the plate boundary (which must be less than the strength of plate boundary $\left.\sigma_{\mathrm{st}}\right)$. As a result, a lower viscosity $\mu_{\mathrm{a}}$ is required to reproduce the same $\sigma_{\text {st }}$, as compared with the results of equation (6) (broken lines, Fig. 8). Setting $\sigma_{\mathrm{R}}\left(\leq \sigma_{\mathrm{st}} \approx 0.78\right.$ $-18 \mathrm{MPa}), \mu_{\mathrm{a}}=10^{19}-10^{20} \mathrm{~Pa} \mathrm{~s}$, and $D_{\mathrm{RS}}=60-140 \mathrm{~km}$, and considering the uncertainty of plate size in plate model, we estimate $\sigma_{\text {st }} \approx 3-75 \mathrm{MPa}$.

In previous studies, the strength of a plate boundary was estimated based on seismic observations, particularly the spatial mapping of earthquake focal mechanisms and their corresponding temporal changes before and after a large earthquake; e.g., Hasegawa et al. (2012) used highresolution data on the approximately 4000 earthquake focal mechanisms in northeast Japan between 2003 and 2011 and found that the 2011 Tohoku earthquake almost completely released the accumulated stress along the plate boundary, and they estimated the stress at release to be as small as 5-15 $\mathrm{MPa}$, suggesting that the presence of water weakened the plate boundary fault. Hardebeck and Hauksson (2001) used the focal mechanism data of approximately 50,000 earthquakes along the San Andreas Fault, mainly between 1981 and 1999, including the 1992 Landers earthquake, and estimated the strength of the fault to be about $20 \pm 10 \mathrm{MPa}$, due in part to the low mechanical strength of smectite (Carpenter et al. 2011). In this study, although the estimated strength of 3-75 MPa may be regarded as a global average strength for a number of plate boundaries of various types, including convergent and transform boundaries, the estimated strength is consistent with a stress level deduced from high-resolution seismic observations of specific areas, as mentioned above.

To naturally reproduce plate-like structures and motions as part of a mantle convection process, including the case of rigid plates with soft plate boundaries, three-dimensional numerical simulations have been used to investigate critical conditions and requirements, especially those concerning rock rheology. Tackley (2000b) and Richards et al. (2001) estimated the required yield stress of a plate to reproduce Earth-like plate motion on the basis of surface velocity fields and obtained results of $17-170$ and $50-150 \mathrm{MPa}$, respectively. In addition, Bercovici (1993, 1995, 2003) suggested that a pseudostick-slip rheology, in which the stress decreases with increasing strain rate after yielding, can reproduce platelike motions, in particular a high toroidal/poloidal kinetic energy ratio of up to 0.8 , which is consistent with ratios observed in previous studies (Bercovici and Wessel 1994; Hager and O'Connell 1978; O'Connell et al. 1991) as well as in this study (Fig. 1). In this context, after exceeding the yield stress of plates, the strain can be concentrated to form a plate boundary composed of a "damaged" weak zone (e.g., Bercovici and Ricard 2014), where the stress level is significantly reduced, possibly to the range estimated from seismology and this study, especially when water is present to weaken the plate boundary. 
The exact rheology and the physical-chemical state of plate boundary is a vital problem that will help understand the mechanisms of plate tectonics (e.g., Bercovici and Ricard 2014; Gordon 1998, 2000). At present, it is difficult to constrain the exact rheology from the approach in this study; however, by combining with other approaches, such as seismic and geodetic observations on both rigid plates and deformation zones (Gordon 1998, 2000; Kreemer et al. 2014), field and laboratory studies on rock and fault rheology (e.g., Kohlstedt et al. 1995; Sibson 2003), and numerical simulation of combined plate motion and mantle convection (e.g., Bercovici 2003; Richards et al. 2001; Tackley 2000b), tighter constraints can be obtained to quantify the Earth's dynamics with regard to plate tectonics.

\section{Conclusion}

The main objective of this study was to constrain the driving forces of plate motion, especially the plate spin motion, for which we analyzed the relationship between toroidal-poloidal components, plate geometry, plate size, spin motion, and their geographical distribution. The following results were obtained. First, a continuous increase in the toroidal/poloidal ratio at high spherical harmonic degrees (>20 up to 1000; Fig. 1) arises from an increase in the spin rate of individual plates (Fig. 4), rather than a consequence of geometrical factors (e.g., a systematic change in aspect ratio with plate size; Fig. 3). Second, spin motion of plates without slabs decreases at plate sizes of $\sim 1000 \mathrm{~km}$ and greater (Fig. 5), which indicates the strength for plate boundaries (Fig. 6). Third, the geographical distribution of spin motion (Fig. 7) suggests that large plates with subducting slabs drive the spin motion of surrounding smaller plates, similar to gears that transmit the shear stress induced by straight motion of large plates. Finally, from the dynamic equilibrium of spin motion at the critical plate size, we obtain the relationship between the strength of the plate boundary and the viscosity of the asthenosphere. Assuming the viscosity and thickness of the asthenosphere to be $10^{19}-10^{20} \mathrm{~Pa} \mathrm{~s}$ and $60-140 \mathrm{~km}$, respectively, we roughly estimated the strength of the plate boundary to be 3-75 MPa, which is comparable to the stress level estimated from several seismological observations, including those in NE Japan associated with the 2011 Tohoku earthquake.

\section{Additional files}

Additional file 1. Brief explanation for reducing calculation time of the spherical harmonic expansion.

Additional file 2: Table S1. List of the plates and parameters for PB2002 in a hotspot reference frame.

\section{Authors' contributions}

TM conducted the quantitative analyses, developed the interpretation, and drafted the manuscript. HI provided the initial idea, developed the interpretation, and improved the manuscript. Both authors read and approved the final manuscript.

\section{Author details}

${ }^{1}$ Department of Earth and Planetary Sciences, Tokyo Institute of Technology, 2-12-1 Ookayama, Meguro-ku, 152-8551 Tokyo, Japan. ${ }^{2}$ Department of Solid Earth Geochemistry, Japan Agency for Marine-Earth Science and Technology (JAMSTEC), 2-15 Natsushima-cho, 237-0061 Yokosuka, Japan.

\section{Acknowledgements}

The digital data for plate configuration used to produce the results of this paper are available for free from the following Web site run by Dr. Peter Bird: http://peterbird.name/publications/2003_PB2002/2003_PB2002.htm. This work was supported in part by the GCOE program [From the Earth to "Earths"].

\section{Competing interests}

The authors declare that they have no competing interests.

Received: 10 December 2015 Accepted: 27 January 2016

Published online: 29 February 2016

\section{References}

Argus DF, Gordon RG, DeMets C (2011) Geologically current motion of 56 plates relative to the no-net-rotation reference frame. Geochem Geophys Geosyst. doi:10.1029/2011GC003751

Beghein C, Trampert J (2004) Probability density functions for radial anisotropy: implications for the upper $1200 \mathrm{~km}$ of the mantle. Earth Planet Sci Lett 217:151-162. doi:10.1016/S0012-821X(03)00575-2

Bercovici D (1993) A simple model of plate generation from mantle convection. Geophys J Int 114:635-650. doi:10.1111/j.1365-246X.1993.tb06993.x

Bercovici D (1995) A source-sink model of the generation of plate tectonics from nonnewtonian mantle flow. J Geophys Res 100:2013-2030. doi:10.1029/94JB02598

Bercovici D (2003) The generation of plate tectonics from mantle convection. Earth Planet Sci Lett 205:107-121. doi:10.1016/S0012-821X(02)01009-9

Bercovici D, Wessel P (1994) A continuous kinematic model of plate-tectonic motions. Geophys J Int 119:595-610. doi:10.1111/j.1365-246X.1994. tb00144.x

Bercovici D, Ricard Y (2014) Plate tectonics, damage and inheritance. Nature 508:513-516. doi:10.1038/nature13072

Bird P (2003) An updated digital model of plate boundaries. Geochem Geophys Geosyst. doi:10.1029/2001GC000252

Cadek O, Ricard Y (1992) Toroidal poloidal energy partitioning and global lithospheric rotation during Cenozoic time. Earth Planet Sci Lett 109:621632. doi:10.1016/0012-821X(92)90120-K

Carpenter BM, Marone C, Saffer DM (2011) Weakness of the San Andreas Fault revealed by samples from the active fault zone. Nature Geosci 4:251-254. doi:10.1038/NGEO1089

Dziewonski A, Anderson D (1981) Preliminary reference earth model. Phys Earth Planet Inter 25:297-356. doi:10.1016/0031-9201(81)90046-7

Evans RL, Hirth G, Baba K, Forsyth D, Chave A, Mackie R (2005) Geophysical evidence from the melt area for compositional controls on oceanic plates. Nature 437:249-252. doi:10.1038/nature04014

Foley BJ, Becker TW (2009) Generation of plate-like behavior and mantle heterogeneity from a spherical, viscoplastic convection model. Geochem Geophys Geosyst. doi:10.1029/2009GC002378

Forsyth D, Uyeda S (1975) On the relative importance of the driving forces of plate motion. Geophys J R Astron Soc 43:163-200. doi:10.1111/j.1365246X.1975.tb00631.x

Forte A, Mitrovica J (2001) Deep-mantle high-viscosity flow and thermochemical structure inferred from seismic and geodynamic data. Nature 410:1049-1056. doi:10.1038/35074000

Gorbatov A, Fukao Y (2005) Tomographic search for missing link between the ancient Farallon subduction and the present Cocos subduction. Geophys J Int 160:849-854. doi:10.1111/j.1365-246X.2005.02507.x 
Gordon RG (1998) The plate tectonic approximation: Plate nonrigidity, diffuse plate boundaries, and global plate reconstructions. Annu Rev Earth Planet Sci 26:615-642. doi:10.1146/annurev.earth.26.1.615

Gordon R (2000) Diffuse oceanic plate boundaries: strain rates, vertically averaged rheology and comparisons with narrow plate boundaries and stable interiors. In: Richards M, Gordon R, van der Hilst R (eds) History and dynamics of global plate motions. AGU, Washington, DC

Gripp A, Gordon R (2002) Young track of hotspots and current plate velocities. Geophys J Int 150:321-361. doi:10.1046/j.1365-246X.2002.01627.x

Hager B, O'Connell R (1978) Subduction zone dip angles and flow driven by plate motion. Tectonophys 50:111-133. doi:10.1016/0040-1951(78)90130-0

Handa S (2005) Electrical conductivity structures estimated by thin sheet inversion, with special attention to the Beppu-Shimabara graben in central Kyushu, Japan. Earth Planets Space 57:605-612. doi:10.1186/ BF03351839

Hardebeck JL, Hauksson E (2001) Crustal stress field in southern California and its implications for fault mechanics. J Geophys Res 106:21,859-21,882. doi :10.1029/2001JB000292

Hasegawa A, Yoshida K, Asano Y, Okada T, linuma T, Ito Y (2012) Change in stress field after the 2011 great Tohoku-Oki earthquake. Earth Planet Sci Lett 355-356:231-243. doi:10.1016/j.epsl.2012.08.042

Karato S (2012) On the origin of the asthenosphere. Earth Planet Sci Lett 321-322:95-103. doi:10.1016/j.epsl.2012.01.001

Karato S, Wu P (1993) Rheology of the upper mantle: a synthesis. Science 260:771-778. doi:10.1126/science.260.5109.771

Kawakatsu H, Kumar P, Takei Y, Shinohara M, Kanazawa T, Araki E, Suyehiro K (2009) Seismic evidence for sharp lithosphere-asthenosphere boundaries of oceanic plates. Science 324:499-502. doi:10.1126/science.1169499

Kohlstedt D, Evans B, Mackwell S (1995) Strength of the lithosphere: constraints imposed by laboratory measurements. J Geophys Res 100:17,587-17,602. doi:10.1029/95JB01460

Kreemer C, Blewitt G, Klein EC (2014) A geodetic plate motion and Global Strain Rate Model. Geochem Geophys Geosyst. doi:10.1002/201 4GC005407

Lenardic A, Richards MA, Busse FH (2006) Depth-dependent rheology and the horizontal length scale of mantle convection. J Geophys Res 111(B07):404. doi:10.1029/2005JB003639

Lithgow-Bertelloni C, Richards M (1993) Toroidal-poloidal partitioning of plate motions since $120 \mathrm{Ma}$. Geophys Res Lett 20:375-378. doi:10.1029/93GL00168

Long MD, van der Hilst RD (2005) Upper mantle anisotropy beneath Japan from shear wave splitting. Phys Earth Planet Inter 151:206-222. doi:10.1016/j.pepi.2005.03.003

Moresi L, Solomatov V (1998) Mantle convection with a brittle lithosphere: thoughts on the global tectonic styles of the Earth and Venus. Geophys J Int 133:669-682. doi:10.1046/j.1365-246X.1998.00521.x
O'Connell R, Gable C, Hager B (1991) Toroidal-poloidal partitioning of lithospheric plate motion. In: Sabadini R, Lambeck K, Boschi E (eds) Glacial isostasy, sea level and mantle rheology. Springer, Netherlands, Dordrecht

Olson P, Bercovici D (1991) On the equipartition of kinetic energy in plate tectonics. Geophys Res Lett 18:1751-1754. doi:10.1029/91GL01840

Peltier WR (1998) Postglacial variations in the level of the sea: implications for climate dynamics and solid-earth geophysics. Rev Geophys 36:603-689. doi:10.1029/98RG02638

Ricard Y, Doglioni C, Sabadini R (1991) Differential rotation between lithosphere and mantle: a consequence of lateral mantle viscosity variations. J Geophys Res 96:8407-8415. doi:10.1029/91 JB00204

Richards M, Yang W, Baumgardner J, Bunge H (2001) Role of a low-viscosity zone in stabilizing plate tectonics: implications for comparative terrestrial planetology. Geochem Geophys Geosyst. doi:10.1029/2002GC000374

Schouten H, Klitgord K, Gallo D (1993) Edge-driven microplate kinematics. J Geophys Res 98:6689-6701. doi:10.1029/92JB02749

Schubert G, Turcotte D, Olson P (eds) (2001) Mantle convection in the earth and planets. Cambridge University Press, New York

Seno T (2000) Why the Philippine Sea plate moves as it does. J Geol Soc Phil 55:105-117

Seno T, Stein S, Gripp AE (1993) A model for the motion of the Philippine Sea plate consistent with NUVEL-1 and geological data. J Geophys Res 98:17,941-17,948. doi:10.1029/93JB00782

Shimoizumi M, Mogi T, Nakada M, Yukutake T, Handa S, Tanaka Y, Utada H (1997) Electrical conductivity anomalies beneath the Western Sea of Kyushu, Japan. Geophys Res Lett 24:1551-1554. doi:10.1029/97GL01542

Sibson RH (2003) Thickness of the seismic slip zone. Bull Seismol Soc Am 93:1169-1178. doi:10.1785/0120020061

Simons M, Hager B (1997) Localization of the gravity field and the signature of glacial rebound. Nature 390:500-504. doi:10.1038/37339

Tackley P (2000a) Self-consistent generation of tectonic plates in timedependent, three-dimensional mantle convection simulations 1. Pseudoplastic yielding. Geochem Geophys Geosyst 1:2000GC000,036

Tackley P (2000b) Self-consistent generation of tectonic plates in timedependent, three-dimensional mantle convection simulations 2. Strain weakening and asthenosphere. Geochem Geophys Geosyst 1:2000GC000,043

van Heck H, Tackley P (2008) Planforms of self-consistently generated plates in 3D spherical geometry. Geophys Res Lett 35(L19):312. doi:10.1029/20 08GL035190

Yingjie Y, Forsyth DW, Weeraratne DS (2007) Seismic attenuation near the east pacific rise and the origin of the low-velocity zone. Earth Planet Sci Lett 258:260-268. doi:10.1016/j.epsl.2007.03.040

Zhong S, Gurnis M, Moresi L (1998) Role of faults, nonlinear rheology and viscosity structure in generating plates from instantaneous mantle flow models. J Geophys Res 103:15,255-15,268. doi:10.1029/98JB00605

\section{Submit your manuscript to a SpringerOpen ${ }^{\circ}$ journal and benefit from:}

- Convenient online submission

- Rigorous peer review

- Immediate publication on acceptance

- Open access: articles freely available online

- High visibility within the field

- Retaining the copyright to your article

Submit your next manuscript at $>$ springeropen.com 